Pacific Journal of Mathematics

DEMPOTENTS IN THE BOUNDARY OF A LIE GROUP 


\title{
IDEMPOTENTS IN THE BOUNDARY OF A LIE GROUP
}

\section{Frank KNOWLeS}

\begin{abstract}
Let $S$ be a locally compact semigroup consisting of a dense connected Lie group $G$ and its boundary $L$; and let $e$ be an idempotent in $L$. This paper is concerned with the proof of three principal results: (1) If $L=G e$ is simply connected, then $S$ is homeomorphic to $G / G_{r}(e) \times G_{r}(e) / G_{l}(e) \times G_{l}(e)^{-}$, where $G_{l}(e)^{-}$ is a connected locally compact group with zero. (2) For any connected Lie group $G$ and closed normal subgroup $H$ such that $G / H$ is simply connected and $H$ is the direct product of the multiplicative group of positive real numbers and a connected compact group, there is a locally compact semigroup $S$ which contains a dense subgroup isomorphic to $G$ whose boundary is a group isomorphic to $G / H$. (3) If $G=V^{*} G_{l}(e)$, for some subspace $V \subset G$, then $G e$ is locally compact if and only if there is a local cross-section to the global orbits of $G$ at $e$.
\end{abstract}

We first establish notation and state a few basic facts that will be used later. It is understood that we are discussing topological groups and semigroups. Hence, unless stated otherwise, homomorphisms are continuous functions and isomorphisms are homeomorphisms. All spaces are Hausdorff. By the boundary of a set $A$, we mean the set $A^{-} \backslash A$, where $A^{-}$is the usual topological closure of $A$. Let $G$ be a group with subsets $V, W \subset G$ such that at least one is a subgroup. If $V \cap W=\{1\}$, and the multiplication map of $G$ restricted to $V \times W$ is a homeomorphism onto $G$, then we write $G=V^{*} W$. This generalization of the semidirect product will be extremely useful.

Let $G$ be a transformation group acting on the left in the space $X$, and let $x \in X$. The left isotropy subgroup of $x,\{g \in G \mid g x=x\}$ is a closed subgroup of $G$ and is denoted by $G_{l}(e) . G_{r}(e)$ is similarly defined if $G$ acts on the right. The $G$-orbit through $x, G x$, is the set $\{g x \mid g \in G\}$ if $G$ acts on the left, and a similar definition is made for right actions. The $\operatorname{map} h: G / G_{l}(x) \rightarrow G x$, defined by $h\left[g G_{l}(x)\right]=g x$ is always a continuous bijection. If $G$ is connected and locally compact, and $G x$ is locally compact, then $h$ is a homeomorphism [3, p. 7]. If, in addition, $G=V^{*} G_{l}(x)$, then $V$ is homeomorphic to $G x$ by the map $v \rightarrow v x$. (Merely note that $V$ is homeomorphic to $G / G_{l}(x)$ via the projection.)

Suppose now that $G$ is a dense connected Lie subgroup of a locally compact semigroup $S$. Let $L$ be the boundary of $G$. $G$ is open in $G^{-}$(since $G$ is locally compact), so $L$ is closed and locally compact. $G$ acts in $S$ on the left and on the right by the semigroup multiplication. If $x \in L$, then $G x$ is homeomorphic to $G / G_{l}(x)$ if $G x$ is closed 
in $L$. If $G x$ is, in addition, simply connected as well as closed, then $G / G_{l}(x)$ is simply connected, and this implies $G_{l}(x)$ is connected [1, p. 59]. (One should note that if a space is simply connected in the usual sense, then it is simply connected in the sense of [1], and, for manifolds, the two definitions are equivalent $[1, \mathrm{p} .44 ; 3, \mathrm{p} .51]$.$) Recall$ that for any semigroup $S, M(S)$ denotes the unique minimal two-sided ideal of $S$, if it exists. If $L=G e$, where $e$ is an idempotent, then we invariably write $H_{L}, H_{R}$, respectively, for $G_{l}(e)$, and $G_{r}(e)$.

In [4], Hofmann completely describes the locally compact groups with zero on his way to describing the locally compact groups with compact boundary. We will rely heavily on his description of certain of these objects, so we will summarize the pertinent facts and definitions. If $S$ is any semigroup and $e$ is a point of $S$ such that $s e=e$, for all $s \in S$, then $e$ is a right zero for $S$. Left zeros are defined similarly. If $S e=e S=\{e\}$, then $e$ is a zero for $S$. For any idempotent $u$ of $S, H(u)$ denotes the maximal subgroup of $S$ containing $u$. If $S$ is a locally compact semigroup consisting of a locally compact group $G$ and a non-isolated point $e \notin G$, then $S$ is a locally compact group with zero. It is immediate that $G=H(1)$ and $e$ is a zero for $S$ [4, p. 22]. If $G$ is connected, then $G$ is isomorphic to $P \times C$, where $P$ is the multiplicative group of positive real numbers and $C$ is a connected compact group [4, pp. 40,49]. Moreover, $S$ is isomorphic to the quotient semigroup $P^{-} \times C /\{0\} \times C$, where $P^{-}$is the multiplicative semigroup of nonnegative real numbers. We identify $P$ with its image in the quotient semigroup $S$, and we denote the zero of $S$ by $e$. It follows that the closure of $P$ in $S, P \cup\{e\}$, is isomorphic to $P^{-}[5$, p. 51].

1. We begin the proofs with two theorems whose proofs are given in $[6,66.310-312]$ and two small lemmas.

THEOREM 1. Let $G$ be a connected locally compact group of finite dimension embedded in a semigroup, and let $e$ be an idempotent in the closure of $G$ such that $G e$ is locally compact. Then $e \in G_{l}(e)^{-}$.

THEOREM 2. Let $G$ be a connected locally compact group embedded in a locally compact semigroup $S$ in such a way that the boundary of $G$ is a single left $G$-orbit $G e$ where $e$ is an idempotent. If $G=$ $V * H_{L}$, for some subspace $V \subset G$, then (i) $H_{L}^{-}=H_{L} \cup\{e\}$, and (ii) the multiplication map of $S$ restricted to $V \times H_{L}^{-}$is a homeomorphism onto $G^{-}$.

LEMMA 3. Let $G$ be a group and let $(\widetilde{G}, p)$ be a group covering of $G$. Suppose that $\widetilde{G}=\widetilde{V} * \widetilde{H}$, where $\widetilde{H}=p^{-1}(H)$ and $H$ is a closed 
subgroup of $G$. Then $G=V * H$, where $p(\widetilde{V})=V$.

Proof. Clearly $G \subset V H$. We show now that the multiplication map, $m: V \times H \rightarrow G$ is one-to-one. Suppose $v_{1} h_{1}=v_{2} h_{2}$. Then there exist $\widetilde{v}_{1}, \widetilde{v}_{2} \in \widetilde{V}$ and $\widetilde{h}_{1}, \widetilde{h}_{2} \in \widetilde{H}$ such that $p\left(\widetilde{v}_{i}\right)=v_{i}$ and $p\left(\widetilde{h}_{i}\right)=h_{i}$, for $i=1,2$. Thus $p\left(\widetilde{v}_{1}\right) p\left(\widetilde{h}_{1}\right)=p\left(\widetilde{v}_{2}\right) p\left(\widetilde{h}_{2}\right)$. This implies, since $p$ is a homomorphism, that $\widetilde{v}_{1} \widetilde{h}_{1}=\widetilde{v}_{2} \widetilde{h}_{2} \widetilde{h}_{3}$, where $\widetilde{h}_{3} \in($ kernel of $p) \subset \widetilde{H}$. Since $\widetilde{G}=\widetilde{V} * \widetilde{H}, \widetilde{v}_{1}=\widetilde{v}_{2}$ and $\widetilde{h}_{1}=\widetilde{h}_{2} \widetilde{h}_{3}$. Consequently, $p\left(\widetilde{v}_{1}\right)=p\left(\widetilde{v}_{2}\right)$ and $p\left(\widetilde{h}_{1}\right)=$ $p\left(\tilde{h}_{2}\right)$. Thus we have $v_{1}=v_{2}$ and $h_{1}=h_{2}$. We have now only to show that $m$ is open. Let $v_{i} h_{i} \rightarrow x \in G$, and let $\left\{v_{i} h_{i}\right\} \subset N$, an evenly covered neighborhood of $x$. Let $\tilde{N}$ be a component of $p^{-1}(N)$. We have $\widetilde{v}_{i} \widetilde{h}_{i} \rightarrow \widetilde{x}$ in $\tilde{N}$, where $p\left(\widetilde{v}_{i} \widetilde{h}_{i}\right)=p\left(\widetilde{v}_{i}\right) p\left(\widetilde{h}_{i}\right)=v_{i} h_{i}$. This implies that $p\left(\widetilde{v}_{i}\right)=v_{i}$ and $p\left(\widetilde{h}_{i}\right)=h_{i}$. There is a $\widetilde{v}$ and $\tilde{h}$ in $\widetilde{G}$ such that $\widetilde{x}=\widetilde{v} \widetilde{h}$, $\widetilde{v}_{i} \rightarrow \widetilde{v}$, and $\widetilde{h}_{i} \rightarrow \widetilde{h}$, since $\widetilde{G}=\widetilde{V} * \widetilde{H}$. Thus, by continuity of $p, v_{i} \rightarrow$ $p(\widetilde{v}), h_{i} \rightarrow p(\widetilde{h})$, and $x=p(\widetilde{v}) p(\widetilde{h})$. This shows that $m$ is open.

LEMMA 4. If $G$ is a connected Lie group and $H$ is a closed normal subgroup of $G$ such that $G / H$ is simply connected, then there is a subspace $V$ of $G$ such that $G=V * H$.

Proof. Let $(\widetilde{G}, p)$ be a simply connected group covering of $G$, and let $p^{-1}(H)=\widetilde{H}$. Since $G / H$ is simply connected, and $G / H$ is homeomorphic to $\widetilde{G} / \widetilde{H}$, it follows that $\widetilde{H}$ is connected [1, p. 59]. Consequently, there is a subspace $\widetilde{V} \subset \widetilde{G}$ such that $\widetilde{G}=\widetilde{V} * \widetilde{H}[3$, p. 135]. By Lemma $3, G=V * H$, where $V=p(\tilde{V})$.

THEOREM 5. Let $G$ be a connected Lie group which is dense in a locally compact semigroup. Suppose that $L$, the boundary of $G$, is a single left G-orbit generated by an idempotent $e$, and let $S=G \cup L$. Then

(i) $H_{\bar{L}}^{-}=H_{L} \cup\{e\}$ is a locally compact group with zero.

(ii) $e G=H(e)$ is a closed topological subgroup of $L$.

(iii) $L=M(S)$.

(iv) $L$ is a group if and only if $H_{L}=H_{R}$.

(v) $S$ is simply connected if and only if $L$ is simply connected.

(vi) If $L$ is simply connected, then $S$ is homeomorphic to $G / H_{R} \times H_{R} / H_{L} \times H_{L}^{-}$, and $H_{\bar{L}}^{-}$is connected.

(vii) Let $L$ be simply connected. Then $S$ is a manifold with boundary if and only if $H_{L}$ is isomorphic to the multiplicative group of positive real numbers; which happens if and only if $\operatorname{dim} L=\operatorname{dim}$ $G-1$.

Proof. (i) $e$ is in $H_{L}^{-}$, by Theorem 1. Since $e$ is a right identity for $L$ and a right zero for $H_{L}^{-}$it follows that $H_{L}^{-} \cap L=\{e\} . \quad H_{L}$ is 
closed in $G$ so $H_{L}^{-}=H_{L} \cup\{e\}$. Thus $H_{L}^{-}$is a locally compact group with zero.

(ii) That $e G$ is an algebraic subgroup of $L$ follows easily from the fact that $e G \subset G e$. Now, $e S=e\left(G^{-}\right)=e(G \cup G e)=e G \cup e G e=e G$. Similarly, $S e=G e$. Thus $e S \cap S e=e G$, and this implies $e G=H(e)$. Clearly, $e S$ and $S e$ are closed sets, thus $e G$ is closed. Since $e G$ is locally compact, a theorem due to Ellis [2] implies that $e G$ is a topological group.

(iii) The argument for (ii) shows that $L$ is an ideal in $S$. If $I$ is any ideal of $S$, then, for $x \in I \cap L$, there exist elements $g$ and $h$ of $G$ such that $x=g e$ and $e g=h e$. Hence $G e x=G e g e=G h e=G e=$ $L \subset I$. Thus $L=M(S)$.

(iv) Assume $H_{L}=H_{R}$. We will show that $e$ is in the center of $S$. Fix $g \in G$. Since $e G \subset G e, g e g^{-1} \in G e$ so $g e g^{-1}=h e$ for some $h \in G$. Now $e=e g g^{-1}=e g e g^{-1}=e h e=e h$. Thus $h \in H_{R}$. This implies $e=$ $h e=g e g^{-1}$ and $e g=g e$. Thus $G e=e G$ and, by (ii), $L$ is a group. Conversely, if $L=M(S)$ is a group, then $e$ is in the center of $S$ so $H_{L}=H_{R}$.

(v) The map $s \rightarrow s e$ is a retraction of $S$ onto $L$, thus if $S$ is simply connected, $L$ is simply connected. Assume now that $L$ is simply connected. By (i) $H_{L}^{-}$is a locally compact group with zero. Since $L$ is simply connected, $G / H_{L}$ is simply connected and so $H_{L}$ is connected. Consequently, $H_{L}=P \times C$, where $P$ is a subgroup of $G$ isomorphic to the multiplicative group of positive real numbers and $C$ is a connected compact subgroup of $G$. Moreover, $P^{-}=P \cup\{e\}$. Let $[1, e]$ denote the closed arc of $P^{-}$that connects 1 to $e$. Define $T:[1, e] \times S \rightarrow S$ by $T(p, s)=s p$, for $p \in[1, e]$, and $s \in S$. $T$ is a deformation retraction of $S$ onto $L$. Thus $S$ is simply connected.

(vi) The argument for (v) shows that $H_{L}$ is connected, thus $H_{\bar{L}}^{-}$ is connected. Using the notation of the proof of $(\mathrm{v})$, define $T^{\prime}:[1, e] \times$ $S \rightarrow S$ by $T^{\prime}(p, s)=p s$. $T^{\prime}$ is a deformation retraction of $S$ onto $e G$ which implies $e G$ is simply connected. Now, the map $f: G \rightarrow e G$, defined by $f(g)=e g$, is a homeomorphism and $H_{R}$ is the kernel of $f$. Thus $H_{R}$ is a closed normal subgroup of $G$. By Lemma 4, there is a subspace $V$ of $G$ such that $G=V * H_{R}$. We will show that the multiplication map, $m: V \times H_{R}^{-} \rightarrow S$ is a homeomorphism onto $S$. First we show that $H_{R}^{-} \cap L=H_{R} e$. Clearly $H_{R} e \subset H_{R}^{-} \cap L$ since the closure of a subgroup is a semigroup, and $L$ is an ideal. Let $x \in H_{R}^{-} \cap L$. Then, for some $g \in G, x=g e$, and $e=e x=e g e=e g$. Thus $g \in H_{R}$, and $x \in H_{R} e$. Since $G=V H_{R}$ and $L=G e=V H_{R} e$, it is clear that $m$ is onto. We show next that $m$ is one-to-one. Since $m$ is one-to-one on $V \times H_{R}$, we have only to show that if $v_{1} h_{1} e=v_{2} h_{2} e$, for $v_{1}, v_{2} \in V$ and $h_{1}, h_{2} \in H_{R}$, then $v_{1}=v_{2}$ and $h_{1} e=h_{2} e$. Let $v_{1} h_{1} e=v_{2} h_{2} e$. Then $v_{1} h_{1}=v_{2} h_{2} h$, where $h \in H_{L} \subset H_{L}$. (Notice that $H_{L} \subset H_{R}$ since, by (i), 


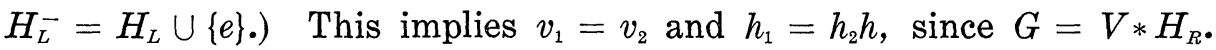
Thus $h_{1} e=h_{2} e$. To show that $m$ is open we have only to show the following two statements: and

(a) $v_{i} h_{i} e \rightarrow v h e \Rightarrow v_{i} \rightarrow v$ and $h_{i} e \rightarrow h e$, where $v_{i} v \in V$ and $h_{i}, h \in H_{R}$;

(b) $v_{i} h_{i} \rightarrow v h e \Rightarrow v_{i} \rightarrow v$ and $h_{i} \rightarrow h_{i} e$, where $v_{i}, v \in V$ and $h_{i}, h \in H_{R}$.

Now, (b) follows easily from (a) (postmultiplication by $e$ ) so we will prove only (a). Let $v_{i} h_{i} e \rightarrow v h e$. Then $e v_{i}=e v_{i} h_{i} e \rightarrow e v h e=e v$. Since $e G$ is a group, this implies $e v_{i}^{-1} \rightarrow e v^{-1}$. Since $G=H_{R} * V^{-1}$, this implies $v_{i}^{-1} \rightarrow v^{-1}$. Thus $v_{i} \rightarrow v$ and $h_{i} e \rightarrow h e$. What has been shown to this point is that $G$ is homeomorphic to $G / H_{R} \times H_{R}^{-}$. In the next paragraph we show that $H_{L}^{-}$is homeomorphic to $H_{R} / H_{L} \times H_{L}^{-}$.

By restricting the maps $T$ and $T^{\prime}$ to $[1, e] \times H_{R}^{-}$, respectively, one sees that $H_{R} e$ is a deformation retraction of $H_{R}^{-}$, and $H_{R}^{-}$is contractible. Hence, $H_{R} e$ is contractible. Since $H_{R} e$ is closed, this implies $H_{R} / H_{L}$ is contractible. Consequently, there is a subspace $W \subset H_{R}$ such that $H_{R}=W * H_{L}[7, \mathrm{p} .53]$. It is worth noticing that $H_{R}^{-}$is a locally compact semigroup with a dense connected Lie group whose boundary is a single left orbit generated by a left zero. A description of these semigroups would be a big step toward completely describing the semigroups we are concerned with in this theorem. We now show that the multiplication map, $m: W \times H_{L}^{-} \times H_{R}^{-}$is a homeomorphism. To show that $m$ is onto, let $k \in H_{R}^{-} \cap L$. We have seen that $H_{R}^{-} \cap L=$ $H_{R} e$. Hence there exists $w \in W, h \in H_{L}$ such that $(w h) e=w e=k$. Since $H_{R}=W * H_{L}$, this shows that $m$ is onto. To show that $m$ is open, we have only to check that $\mathrm{w}_{i} h_{i} \rightarrow w e$, where $w_{i}, w \in W$ and $h_{i} \in H_{L} \Rightarrow w_{i} \rightarrow w$ and $h_{i} \rightarrow e$. If $w_{i} h_{i} \rightarrow w e$, then $w_{i} e \rightarrow w e$. Since $W$ is homeomorphic to We via the map $w \rightarrow w e$, we have $w_{i} \rightarrow w$ and thus $h_{i} \rightarrow e$.

(vii) We saw above that $V \times H_{\vec{R}}^{-}$is homeomorphic to $S$ and $W \times H_{\bar{L}}^{-}$is homeomorphic to $H_{R}^{-}$; each by a suitable restriction of the multiplication map of $S$. Thus $S$ is homeomorphic to $U \times H_{\bar{L}}^{-}$by a restriction of the multiplication map of $S$, where $U=V W \subset G$. Moreover, $G=U * H_{L}$, and $L=U e$ is homeomorphic to $U$. Since $U, P, C$ and $L$ are manifolds, we will mean by the dimension of a space its dimension as a manifold. If $H_{\bar{L}}^{-}=P^{-}$, then, clearly, $S$ is a manifold with boundary. Also, in this case, $\operatorname{dim} G=\operatorname{dim} U+1$. Thus $\operatorname{dim} L=$ $\operatorname{dim} G-1$. We will show now that if $C \neq\{1\}$, then $S$ cannot be a manifold with boundary, and, moreover, $\operatorname{dim} L \leqq \operatorname{dim} G-2$. This suffices to show the equivalence of the three statements of the theorem. It might be well to keep in mind that $S$ could be a manifold without boundary if, say, $C$ were the circle group; for then $H_{\bar{L}}^{-}$would be the multiplicative semigroup of complex numbers.

Let $C \neq\{1\}$. Then $\operatorname{dim} G=\operatorname{dim} U+1+\operatorname{dim} C$. Since $\operatorname{dim} C \geqq 1$ 
( $C$ is connected), we have $\operatorname{dim} L=\operatorname{dim} U \leqq \operatorname{dim} G-2$. We have only to show now that $S$ cannot be a manifold with boundary. We assume that $S$ is a manifold with boundary and arrive at a contradiction. $G$ is an open homogeneous subset of $S$, hence $G \leqq$ Int $S$. Consequently, $\phi \neq b d S \subset L . \quad$ Let $u_{1} e, u_{2} e \in L . \quad U$ is a homogeneous space so there is a homeomorphism $h: U \rightarrow U$ and $h\left(u_{1}\right)=u_{2}$. Let $x \in U, y \in H_{L}^{-}$. The following composition of homeomorphisms is a homeomorphism of $S$ onto $S$ that carries $u_{1} e$ to $u_{2} e: x y \in S \rightarrow(x, y) \in U \times H_{L}^{-} \rightarrow(h(x), y) \in U \times$ $H_{L}^{-} \rightarrow h(x) y \in S$. This implies that all of $L$ must be in the boundary of $S$ and thus $L=b d S$. Consequently, we have the four statements, (i) $L=b d S$, (ii) $G=\operatorname{Int} S$, (iii) $\operatorname{dim} L \leqq \operatorname{dim} G-2$ and (iv) $S$ is a manifold with boundary. This is impossible.

THEOREM 6. Let $G$ be a connected Lie group with a closed normal subgroup $H$ such that $G / H$ is simply connected. Let $H=C \times P$ where $C$ is a connected compact group and $P$ is the multiplicative group of positive real numbers. Then $G$ can be embedded in a locally compact semigroup as a dense subgroup whose boundary is a group isomorphic to $G / H$.

Proof. Let $H^{-}$denote the locally compact semigroup $P^{-} \times C /\{0\} \times C$, where $P^{-}$is the multiplicative semigroup of nonnegative real numbers, and $P=P^{-} \backslash\{0\}$. We identify $P$ with its image in $H^{-}$, and we denote the zero of $H^{-}$by $e$. The closure of $P$ in $H^{-}, P \cup\{e\}$, is isomorphic to $P^{-}$. By Lemma 4 we have $G=V * H$. For $g \in G$, there exist unique $v \in V, h \in H$ such that $g=v h$. We define $[g]$ to be $v$. Since $H$ is normal and multiplication in $G$ is associative, we have $\left[g_{1}\left[g_{2} g_{3}\right]\right]=$ [[ $\left.\left.g_{1} g_{2}\right] g_{3}\right] . \quad$ Also, $\left[g_{1} g_{2}\right]=\left[v_{1} v_{2}\right]$ where $g_{1}=v_{1} h_{1}$ and $g_{2}=v_{2} h_{2}$; and $g_{i} \rightarrow$ $g \Longrightarrow\left[g_{i}\right] \rightarrow[g]$.

Consider the function $m:\left(V \times H^{-}\right) \times\left(V \times H^{-}\right) \rightarrow V \times H^{-}$defined by (i) $m$ restricted to $(V \times H) \times(V \times H)$ is the multiplication of $G$. (ii) $\left.m\left(\left(v_{1}, e\right),\left(v_{2}, h\right)\right)\right)=m\left(\left(\left(v_{1}, h\right),\left(v_{2}, e\right)\right)\right)=m\left(\left(\left(v_{1}, e\right),\left(v_{2}, e\right)\right)\right)=\left(\left[v_{1} v_{2}\right], e\right)$. It is straightforward to show that $m$ is an associative multiplication. To show that $m$ is a continuous multiplication on $V \times H^{-}$it suffices to show the following statements:

(1) $\left(v_{i}, e\right) \rightarrow(v, e)$ and $\left(v_{1 i}, h_{i}\right) \rightarrow\left(v_{1}, h_{1}\right) \Rightarrow\left(\left[v_{i} v_{1 i}\right], e\right) \rightarrow\left(\left[v v_{1}\right], e\right)$ and $\left(\left[v_{1 i} v_{i}\right], e\right) \rightarrow\left(\left[v_{1} v\right], e\right)$.

(2) $\left(v_{i}, e\right) \rightarrow(v, e)$ and $\left(v_{1 i}, h_{i}\right) \rightarrow\left(v_{1}, e\right) \Rightarrow\left(\left[v_{i} v_{1 i}\right], e\right) \rightarrow\left(\left[v v_{1}\right], e\right)$ and $\left(\left[v_{1 i} v_{i}\right], e\right) \rightarrow\left(\left[v_{1} v\right], e\right)$.

(3) $\left(v_{i}, e\right) \rightarrow\left(v_{1}, e\right)$ and $\left(v_{i}^{\prime}, e\right) \rightarrow\left(v^{\prime}, e\right) \Rightarrow\left(\left[v_{i} v_{i}^{\prime}\right], e\right) \rightarrow\left(\left[v v^{\prime}\right], e\right)$.

(4) $\left(v_{1 i}, h_{1 i}\right) \rightarrow\left(v_{1}, h_{1}\right)$ and $\left(v_{2 i}, h_{2 i}\right) \rightarrow\left(v_{2}, e\right) \Rightarrow\left(\left[v_{1 i} v_{2 i}\right], h_{i}^{\prime \prime} h_{i}^{\prime} h_{2 i}\right) \rightarrow\left(\left[v_{1} v_{2}\right], e\right)$ where $h_{i}^{\prime}=v_{2 i}^{-1} h_{1 i} v_{2 i}$ and $v_{1 i} v_{2 i}=\left[v_{1 i} v_{2 i}\right] h_{i}^{\prime \prime}$.

(5) $\left(v_{1 i}, h_{1 i}\right) \rightarrow\left(v_{1}, e\right)$ and $\left(v_{2 i}, h_{2 i}\right) \rightarrow\left(v_{2}, h_{2}\right) \Rightarrow\left(\left[v_{1 i} v_{2 i}\right], h_{i}^{\prime \prime} h_{i}^{\prime} h_{2 i}\right) \rightarrow\left(\left[v_{1} v_{2}\right], e\right)$ where $h_{i}^{\prime}=v_{2 i}^{-1} h_{1 i} v_{2 i}$ and $v_{1 i} v_{2 i}=\left[v_{1 i} v_{2 i}\right] h_{i}^{\prime \prime}$. 
(6) $\left(v_{1 i}, h_{1 i}\right) \rightarrow\left(v_{1}, e\right)$ and $\left(v_{2 i}, h_{2 i}\right) \rightarrow\left(v_{2}, e\right) \Rightarrow\left(\left[v_{1 i} v_{2 i}\right], h_{i}^{\prime \prime} h_{i}^{\prime} h_{2 i}\right) \rightarrow\left(\left[v_{1} v_{2}\right], e\right)$ where $h_{i}^{\prime}=v_{2 i}^{-1} h_{1 i} v_{2 i}$ and $v_{1 i} v_{2 i}=\left[v_{1 i} v_{2 i}\right] h_{i}^{\prime \prime}$.

(1)-(4) are immediate, and (5)-(6) will follow if we show:

(*) $v_{i} \rightarrow v$ and $h_{i} \rightarrow e \Rightarrow v_{i} h_{i} v_{i}^{-1} \rightarrow e$, where $v_{i} \in V, h_{i} \in H$. We now show $(*)$.

For each $g \in G$, let $A g$ be the automorphism of $H$ induced by the inner automorphism of $G, x \rightarrow g x g^{-1}$. Let $k$ be the projection of $H$ onto $P$, and let $k A g \mid P$ be denoted by $k g$. Notice that $C$ is the maximal compact subgroup of $H$, so $C$ is invariant under $A g$ for all $g \in G$. Clearly, for any $g \in G, k g$ is an automorphism of $P$. It follows from this that the map $g \rightarrow k g$ is a continuous homomorphism of $G$ into the connected component of the identity of the automorphism group of $P$. We assume now that $P$ is the group of additive reals. Thus $k g \in A(P)_{0}$, which we identify with the multiplicative positive reals. We may assume that $p_{i} \rightarrow e$ in $H$ if and only if $p_{i} \rightarrow+\infty$ in the usual orientation of $P$. Thus, for each $g \in G$, there is a positive real number $a(g)$ such that $k g(p)=a(g) \cdot p$ for all $p \in P$. Consequently, $p_{i} \rightarrow e$ if and only if $k g\left(p_{i}\right) \rightarrow e$ for all $g \in G$. Consider $\left\{c_{i} p_{i}\right\} \subset H$, where $c_{i} \in C$ and $p_{i} \in P$. Let $A g\left(c_{i} p_{i}\right)=c_{i 1} c_{i}^{\prime} p_{i}^{\prime}$, where $A g\left(c_{i}\right)=c_{i 1}$ and $A g\left(p_{i}\right)=c_{i}^{\prime} p_{i}^{\prime} . \quad$ Clearly, $A g\left(c_{i} p_{i}\right) \rightarrow e \Leftrightarrow p_{i}^{\prime} \rightarrow e \Leftrightarrow k g\left(p_{i}\right) \rightarrow e \Leftrightarrow p_{i} \rightarrow e \Leftrightarrow$ $c_{i} p_{i} \rightarrow e$.

Now let $v_{i} \rightarrow v, v_{i}, v \in V$, and $h_{i}=c_{i} p_{i} \rightarrow e$. Then $v_{i}\left(c_{i} p_{i}\right) v_{i}^{-1}=$ $c_{i}^{\prime}\left(a\left(v_{i}\right) \cdot p_{i}\right)$, so $v_{i}\left(c_{i} p_{i}\right) v_{i}^{-1} \rightarrow e \Leftrightarrow a\left(v_{i}\right) \cdot p_{i} \rightarrow e$. Since $v_{i} \rightarrow v$ and $p_{i} \rightarrow \infty$, it follows that $a\left(v_{i}\right) \rightarrow a(v)$, and thus $a\left(v_{i}\right) \cdot p_{i} \rightarrow \infty$. Thus $v_{i} h_{i} v_{i}^{-1} \rightarrow e$, and we are through.

Clearly, $V \times H^{-}$is locally compact and contains a dense subgroup isomorphic to $G$. The map $f: G \rightarrow V \times\{e\}$ defined by $f(g)=([g], e)$ induces an isomorphism from $G / H$ onto $V \times\{e\}$, the boundary of $V \times H$ in $V \times H^{-}$.

REMARKS AND ExAmples. If $G$ is an abelian Lie group, then it is not too difficult to see that we may pick $V$ to be a vector group and that $S$ is the product semigroup $V \times H^{-}$. In this case, our result is a corollary of a theorem of Stepp's [8, Theorem 3, p. 404]. The semigroups on a three-dimensional half-space without radical [5, pp. 45-48] provide a variety of examples of semigroups that satisfy the hypotheses of Theorem 5. It is worth noticing here that if $G$ is the noncommutative nilpotent group on $E^{3}$ and $S$ is the half-space semigroup $G \cup G / P$, where $P$ is the center of $G$, then $P$ has no complementary group in $G$ [6]. In [5, pp. 46-47] Horne constructs in a completely different way a half-space semigroup $G \cup G / P$, where the boundary of $G$ is the group $G / P$ for any group $G$ on $E^{3}$ which has a normal one-parameter subgroup $P$. It is clear that the construction given in the argument of Theorem 6 will provide an $n$-dimensional 
half-space semi-group for any group on $E^{n}$ which has a normal oneparameter subgroup.

Our next result is a theorem concerning the action of $G$ at $e$ that is an improvement of Theorem 2. Following Definition 1.10, page 315 (and the Remark on the page) of Hofmann and Mostert's book, Elements of Compact Semigroups [Charles E. Merrill Books, Inc. (1966)], we will define a local cross section to the local orbits and a local cross section to the global orbits of a transformation group at a point. For connected locally compact groups $G$ of finite dimension action on completely regular spaces $X$, our definition of a local cross section to the local orbits is equivalent to that given in Hofmann and Mostert's book.

DEFINITION Let $G$ be a transformation group acting on the right on a space $X$. Let $x \in X$, and let $H=G_{r}(x)$. We call the triple $(C, K, U)$ a local cross section to the local orbits of $G$ at $x$ if

(1) $U$ is a neighborhood of $x$;

(2) $C$ is a closed subset of $U$ containing $x$;

(3) $K$ is a closed subset of $G$ containing 1 that is mapped homeomorphically, via the projection map, onto a neighborhood of $H$ in $G / H=\{H g \mid g \in G\}$;

(4) the action of $G$, restricted to $C \times K$, is a homeomorphism onto $U$.

If $G$ acts on $X$ on the left, then $H=G_{l}(x)$, and $(C, K, U)$ is a local cross section to the local orbits of $G$ at $x$ where $C, K$ are as above except that $G / H=\{g H \mid g \in G\}$ in (3), and $K \times C$ is the domain of the action in (4). If, in addition to the above, $K$ maps onto $G / H$ in (3), then $(C, K, U)$ is a local cross section to the global orbits of $G$ at $x$. If then $U=X$, we have a global or complete cross section to the action of $G$ at $x$.

THEOREM 7. Let $G$ be a connected, locally compact, dense subgroup of a locally compact semigroup $S$. Let $e$ be an idempotent in the closwie of $G$ such that $G=W * H$, where $H$ is the left isotropy subgroup of e relative to the natural action of $G$ on $S$ by the multiplication in $S$. It follows that $G e$ is a locally compact subspace of $S$ if and only if $\left(H^{-}, W, W H^{-}\right)$is a local cross section to the global orbits of $G$ at $e$.

Proof. Assume Ge locally compact. Then $W$ is homeomorphic to $W e=G e$ via the map $w \rightarrow w e$. There is a net $w_{i} h_{i} \rightarrow e$, so $w_{i} e \rightarrow e$ and $w_{i} \rightarrow 1$. This implies $h_{i} \rightarrow e$ and $e \in H^{-}$. We now show that the multiplication map, $W \times H^{-} \rightarrow W H^{-}$is a homeomorphism. If $w_{1} h_{1}=$ $w_{2} h_{2}$, where $w_{i} \in W, h_{i} \in H^{-}$, then $w_{1} e=w_{2} e w_{1}=w_{2}$. Thus $h_{1}=h_{2}$ 
and $m$ is one-to-one. Suppose $w_{i} h_{i} \rightarrow w h$, where $w_{i}, w \in W$ and $h_{i}, h \in H^{-}$. Then $w_{i} e \rightarrow w e$, so $w_{i} \rightarrow w$. Hence $h_{i} \rightarrow h$ and $m$ is open onto $W H^{-}$. We have show that $m: W \times H^{-} \rightarrow S$ is a homeomorphism into $S$. Since $e \in H^{-}, G \cup G e \subset W H^{-}$. Since $W \times H^{-}$is locally compact, $W H^{-}$ is a locally compact, dense subset of $S$. Thus $W H^{-}$is an open subset of $S$.

Assume that $\left(H^{-}, W, W H^{-}\right)$is a local cross section to the global orbits of $G$ at $e$. It is immediate that $G \subset W H$ and $e \in H^{-}$, so $G e=$ $m(W \times\{e\}$ is locally compact since $W \times\{e\}$ is locally compact.

We conclude with a corollary that has applications to half-space semigroups. First, we mention a fact that will be used in the proof that follows. A reference for the relevant theorems about Lie groups is H. Samelson's survey article, Topology of Lie groups [Bull. Amer. Math. Soc., 58 (1952), 2-37]. Let $G$ be a connected Lie group and let $H$ be a maximal compact subgroup of $G$. A theorem of Iwasawa states that $G$ is homeomorphic to the product space $H \times V$, where $V$ is a euclidean space. Since $H$ is an orientable manifold and consequently not acyclic, it follows that $G$ is a group on euclidean space if and only if $G$ contains no nontrivial compact subgroup. An immediate consequence of this is that a closed connected subgroup of a group on euclidean space is a group on euclidean space.

Corollary 8. Let $G$ be a lie group on euclidean $n$-space and a dense subgroup of a locally compact semigroup $S$. Let e be an idempotent in the boundary of $G$ such that $G e$ is a locally compact and simply connected subspace of $S$. Then $G=W * H$, for some $W \subset G$, where $H$ is the left isotropy subgroup of e relative to the natural action of $G$ on $S$ by the multiplication in $S$. Consequently, $\left(H^{-}, W\right.$, $\left.W H^{-}\right)$is a local cross section to the global orbits of $G$ at $e$.

Proof. Since $G e$ is locally compact, $G e$ is homeomorphic to $G / H$, so $G / H$ is simply connected. This implies that $H$ is connected. By the remarks above, $H$ is a group on euclidean $m$-space, $m \leqq n$. Since $G$ is a bundle over $G / H$ with solid fibre $H$, there is a cross section, $f: G / H \rightarrow G[7$, p. 55]. Let $f(G / H)=W$. Clearly $p \mid W$ is the inverse of $f$, so $f$ is a homeomorphism. If now, $w_{i} h_{i} \rightarrow w h$, where $w_{i}, w \in W$, $h_{i}, h \in H$, then $p\left(w_{i}\right) \rightarrow p(w)$ which implies $w_{i} \rightarrow w$. Thus $h_{i} \rightarrow h$ and we have shown that the multiplication map of $G$, restricted to $W \times H$ is open onto $W H=G$. Clearly this map is also injective and continuous, so $G=W^{*} H$. Theorem 7 implies now that $\left(H^{-}, W, W H^{-}\right)$ is a local cross section to the global orbits of $G$ at $e$. 


\section{REFERENCES}

1. C. Chevalley, Theory of Lie groups, Princeton University Press, 1946.

2. R. Ellis, $A$ note on the continuity of the inverse, Proc. Amer. Math. Soc., 8 (1957), 372-373.

3. G. Hochschild, The Structure of Groups, Holden-Day, Inc., 1965.

4. K. Hofmann, Locally compact semigroups in which a subgroup with compact complement is dense, Trans. Amer. Math. Soc., 106 (1963), 19-51.

5. J. G. Horne, Semigroups on a half-space, Trans. Amer. Math. Soc., 147 (1970), 1-53.

6. F. Knowles, Semigroups that are the union of a group on $E^{3}$ and a plane, Trans. Amer. Math. Soc., 160 (1971), 305-325.

7. N. Steenrod, The Topology of Fibre Bundles, Princeton University Press, 1951.

8. J. W. Stepp, D-semigroups, Proc. Amer. Math. Soc., 22 (1969), 402-406.

Received March 3, 1971 and in revised form July 28, 1972. Thanks are due to the referee who pointed out several errors in the original manuscript and whose suggestions have resulted in more readable arguments, particularly those for Theorems 5 and 7.

WAYNe State UNIVERSITY 


\section{PACIFIC JOURNAL OF MATHEMATICS}

\section{EDITORS}

H. SAMELSON

Stanford University

Stanford, California 94305

C. R. Hовву

University of Washington

Seattle, Washington 98105
J. DugundJI

Department of Mathematics University of Southern California

Los Angeles, California 90007

RICHARD ARENS

University of California

Los Angeles, California 90024

\section{ASSOCIATE EDITORS}

E. F. BECKENBACH

B. H. NeumanN

F. WOLF

K. YoSHIDA

\section{SUPPORTING INSTITUTIONS}

UNIVERSITY OF BRITISH COLUMBIA

CALIFORNIA INSTITUTE OF TECHNOLOGY

UNIVERSITY OF CALIFORNIA

MONTANA STATE UNIVERSITY

UNIVERSITY OF NEVADA

NEW MEXICO STATE UNIVERSITY

OREGON STATE UNIVERSITY

UNIVERSITY OF OREGON

OSAKA UNIVERSITY
UNIVERSITY OF SOUTHERN CALIFORNIA

STANFORD UNIVERSITY

UNIVERSITY OF TOKYO

UNIVERSITY OF UTAH

WASHINGTON STATE UNIVERSITY

UNIVERSITY OF WASHINGTON

AMERICAN MATHEMATICAL SOCIETY

NAVAL WEAPONS CENTER

Printed in Japan by International Academic Printing Co., Ltd., Tokyo, Japan 


\section{Pacific Journal of Mathematics}

\section{Vol. 44, No. $1 \quad$ May, 1973}

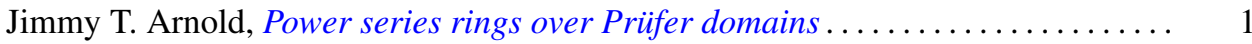

Maynard G. Arsove, On the behavior of Pincherle basis functions . . . . . . . . . 13

Jan William Auer, Fiber integration in smooth bundles ................. 33

George Bachman, Edward Beckenstein and Lawrence Narici, Function algebras

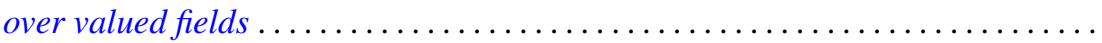

Gerald A. Beer, The index of convexity and the visibility function . . . . . . . . . . .

James Robert Boone, A note on mesocompact and sequentially mesocompact

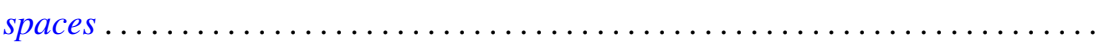

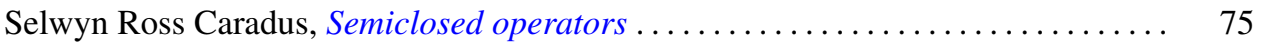

John H. E. Cohn, Two primary factor inequalities . . . . . . . . . . . . . . . 81

Mani Gagrat and Somashekhar Amrith Naimpally, Proximity approach to

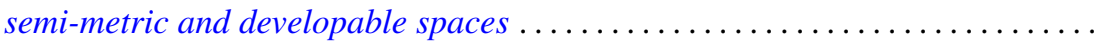

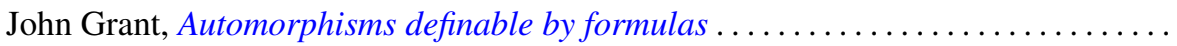

Walter Kurt Hayman, Differential inequalities and local valency ..............

Wolfgang H. Heil, Testing 3-manifolds for projective planes . . . . . . . . . . . . .

107

Melvin Hochster and Louis Jackson Ratliff, Jr., Five theorems on Macaulay

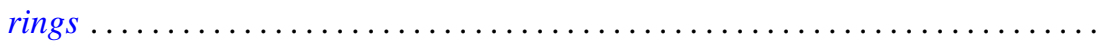

Thomas Benton Hoover, Operator algebras with reducing invariant subspaces ....

James Edgar Keesling, Topological groups whose underlying spaces are separable

Fréchet manifolds...

Frank Leroy Knowles, Idempotents in the boundary of a Lie group . .

191

George Edward Lang, The evaluation map and EHP sequences ...

201

Everette Lee May, Jr, Localizing the spectrum . . . . . . . . . . . .

211

Frank Belsley Miles, Existence of special $K$-sets in certain locally compact abelian groups.

Susan Montgomery, A generalization of a theorem of Jacobson. II . .

T. S. Motzkin and J. L. Walsh, Equilibrium of inverse-distance forces in

three-dimensions.

Arunava Mukherjea and Nicolas A. Tserpes, Invariant measures and the converse

of Haar's theorem on semitopological semigroups .

James Waring Noonan, On close-to-convex functions of order $\beta$

Donald Steven Passman, The Jacobian of a growth transformation

Dean Blackburn Priest, A mean Stieltjes type integral ........ .

Joe Bill Rhodes, Decomposition of semilattices with applications to topological

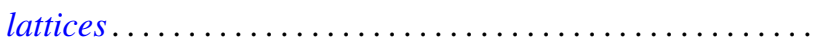

Claus M. Ringel, Socle conditions for $\mathrm{QF}-1$ rings ..........

Richard Rochberg, Linear maps of the disk algebra

Roy W. Ryden, Groups of arithmetic functions under Dirichlet convolution . .

Michael J. Sharpe, A class of operators on excessive functions

Erling Stormer, Automorphisms and equivalence in von Neumann algebras ..

Philip C. Tonne, Matrix representations for linear transformations on series

analytic in the unit disc. 